\title{
The Number Continuity Service: Part II - GSM <-> CDMA Seamless Technology Change
}

\author{
Arnaud Henry-Labordère \\ HALYS, Paris, France and PRISM-CNRS, Versailles, France; e-mail: ahl@halys.fr
}

Received 7 May 2013; Accepted 15 May 2013

\begin{abstract}
Even with the predominance of the GSM technology, there are instances when the only coverage for a GSM outbound subscriber is from a CDMA (IS-41) network (that includes many fixed networks in the USA and the CDMA based Globalstar satphone services). Symmetrically a CDMA subscriber roaming in a GSM network after changing for a GSM handset, will need the number continuity service to receive his calls and SMS. Roaming Hubs able to convert the two signalling systems are required. We explain the differences into the mobility protocols (MAP GSM and MAP IS-41), the TCAP protocol (ANSI and ITU) and the network layers (14 bits ITU and 24 bits ANSI Point Codes). The eight possible combinations are handled by a multi-standard Roaming Hub architecture. Detailed protocol traces are included.
\end{abstract}

Keywords: MAP GSM, MAP IS-41, GSM-CDMA converter, ITU, ANSI.

\section{History and GSM $<->$ CDMA Protocols Comparison}

In 2004, a CDMA<-> GSM number continuity service was provided by Worldcell (USA), mainly for government officials having a CDMA number phone. When they were going abroad, there was no roaming possible,

Journal of Cyber Security and Mobility, Vol. 2, 83-103.

(C) 2013 River Publishers. All rights reserved. 
and they had a GSM phone with many roaming agreements rented for this purpose. The "Number continuity" platform developed by Logica, allowed them to receive calls and SMS on their usual US number, and when they were making calls or SMS and their usual US number was appearing as CLI. Since then the platform was sold, but it is not maintained and is then no longer operational. A number continuity project with Globalstar gave a strong reason to redevelop the technology using a more modern Roaming Hub platform, as Globalstar has two types of core networks and terminals (GSM with an Alcatel HLR in Toulouse, and IS-41 (CDMA) with a DSC HLR in Texas.

It is the same system which would allow for example an ordinary CDMA subscriber (example SPRINT in the US) to visit Russia, rent a GSM phone if his own handset is not bi-standard (such as certain Iphones), and get a local IMSI. If this HPLMN has a CDMA $<->$ GSM roaming hub, he would have the full number continuity service. The CDMA $<->$ GSM Hub is still useful as there are many ( $>10 \%$ of the world mobile users) CDMA networks, in the US (notably SPRINT, Verizon, Metro PCS, Cricket), Asia and Africa (the reason being that the CDMA operators' licences and the core networks are much cheaper). The user changes technology either by getting an other handset or with a mutlti-standard handset (some Iphone versions have the two modes). There exist GSM networks in North America (ANSI) and Europe (ITU), CDMA networks in North America (ANSI) and Europe, Africa or Asia (ITU). Table 1 presents the differences between ANSI and ITU networks whether they are CDMA or GSM.

\subsection{TCAP ITU and TCAP ANSI Comparison}

However, most GSM networks (T-Mobile USA, AT\&T, Canadian GSM) use TCAP ITU even if they are in an ANSI area.

The TCAP ANSI and ITU look similar but are not compatible. It is not just a simple matter of changing the TCAP operation codes, the Component codes and the Transaction Ids also need to be changed. So if interworking needs to be performed between two networks, one with TCAP ANSI, the other TCAP ITU, the Roaming Hub needs two TCAP instances running in parallel. 


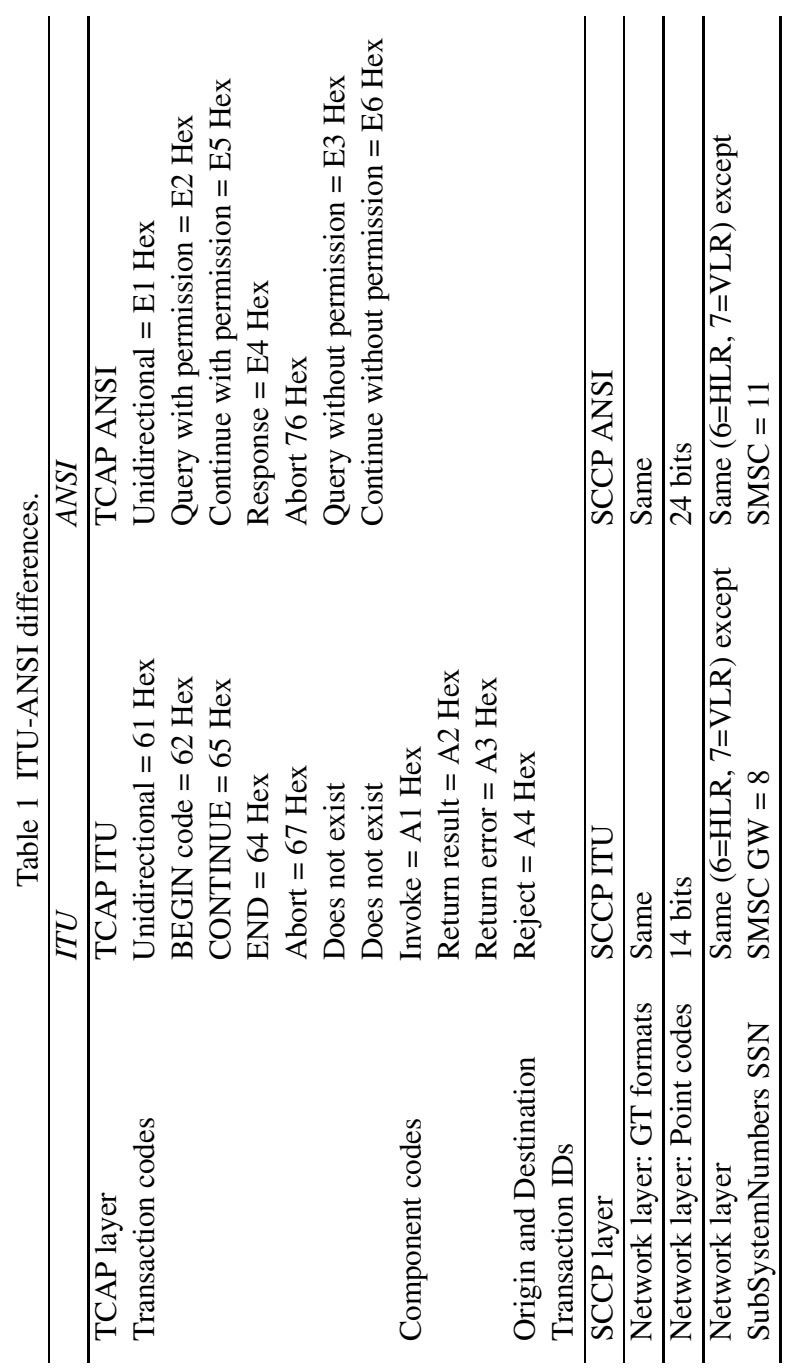




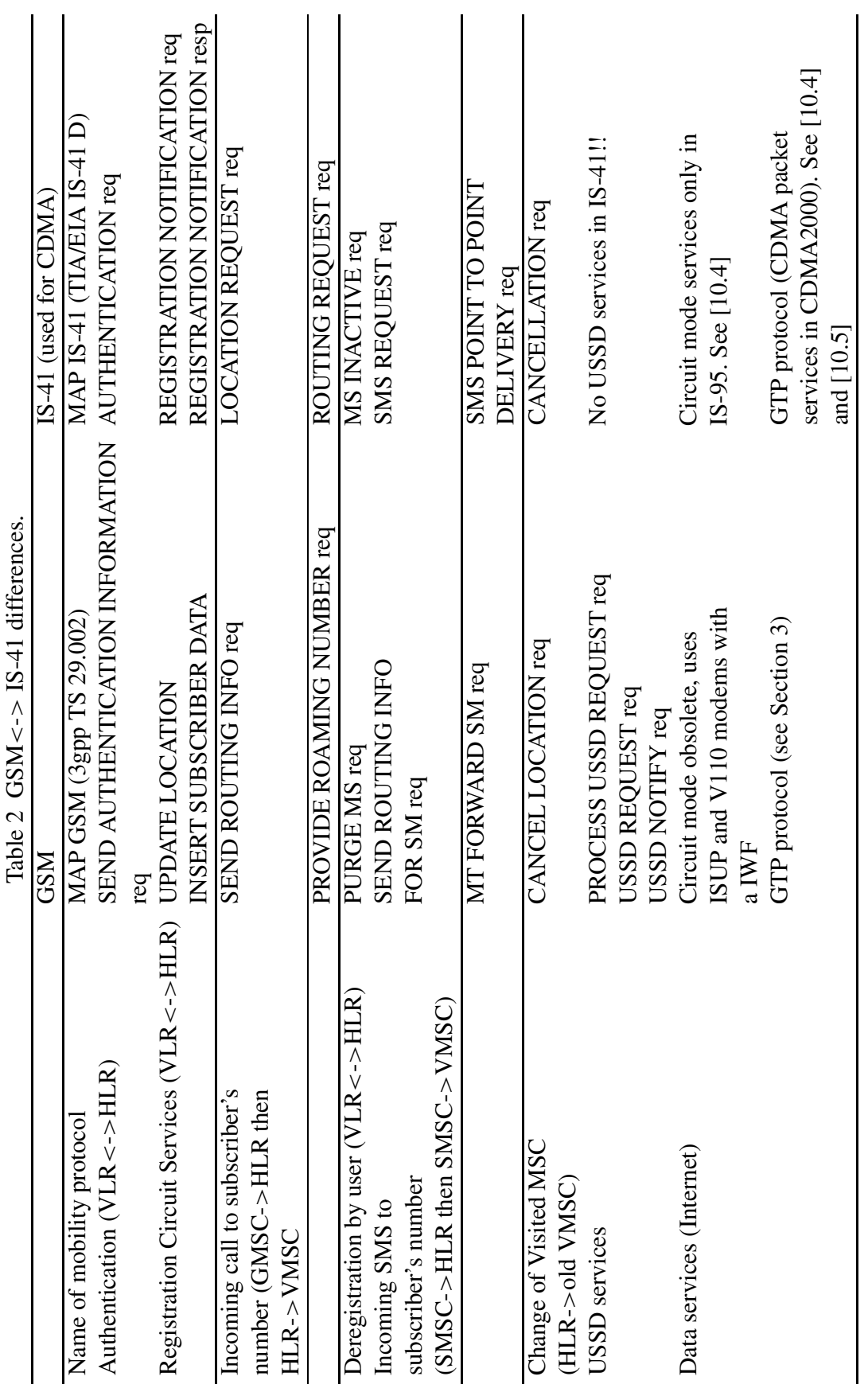


The Number Continuity Service. Part II 87

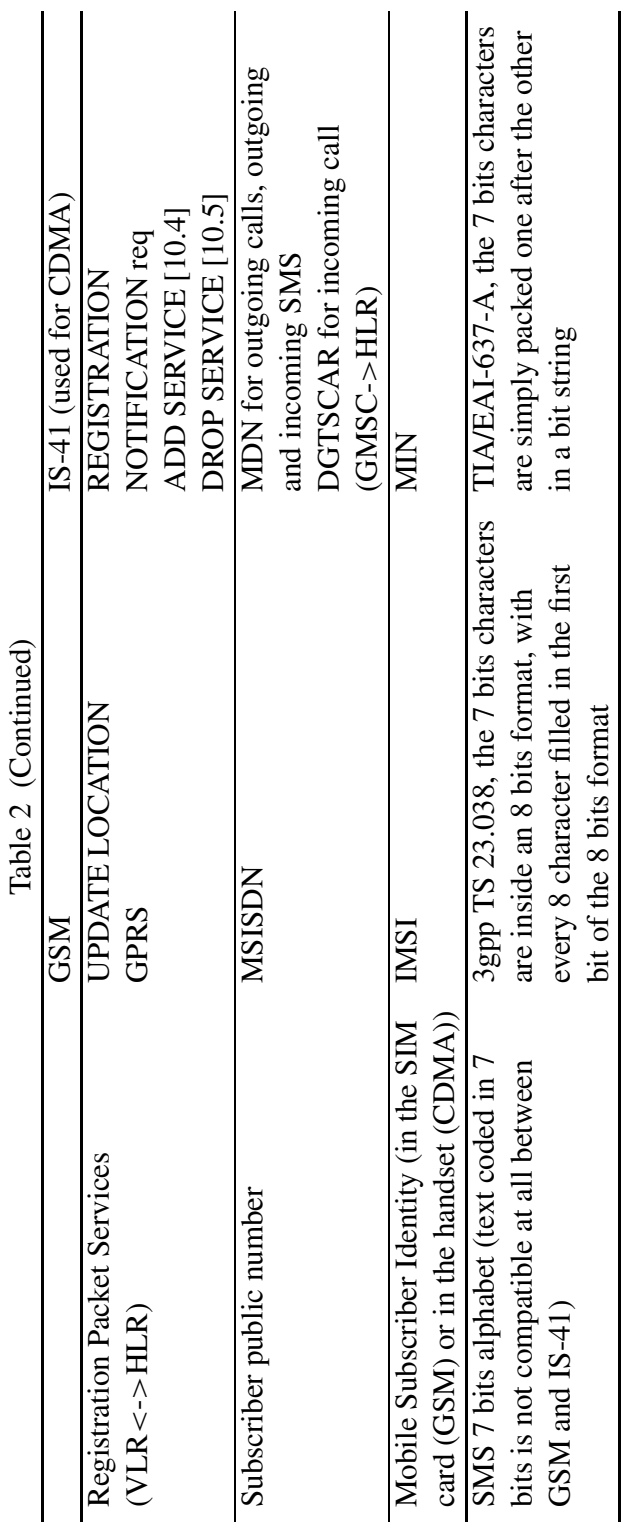




\subsection{MAP GSM and MAP IS-41 Comparison}

Both mobility protocols are called MAP. Table 2 lists the most striking differences. We have given the full list regarding the "number continuity service", including voice and SMS services.

As a consequence of the SSN (Sub System Number) being the same in GSM and IS-41, and of a common international gateway being used by a service provider of number continuity, a routing of the incoming traffic to the MAP GSM stack or the MAP IS-41 stack, cannot be based on the SSN as in most network equipments software (146 goes to Camel, the others to MAP).

For a general operation there must be two routing levels based on a table of Global Titles (GT) specifying the ANSI networks (GSM or CDMA) (so the incoming traffic is sent to TCAP ANSI or the TCAP ITU), and after the TCAP layer, a table specifying the MAP IS-41 or the MAP GSM. A diagram is given in Figure 3.

Such a mixed GSM $<->$ CDMA roaming platform is then much more complex than the implementations which have appeared in the past years, as they necessitate a non standard SS7 architecture using ITU, ANSI, GSM, IS-41 components with some non standard routing levels between the layers. The details given below are for those who want to develop or just need to understand how it works.

To simplify a little we have assumed that the GSM $<->$ CDMA Hub is connected to an ITU SS7 provider which is offering the ANSI $<->$ ITU Point Code conversion with a partner in the path to the ANSI networks. This is why we see a single MTP3 and M3UA layer as well as a single SCCP ITU layer. If there is no ANSI $<->$ ITU Point Code conversion, it is possible to run with two instances of SCCP and two instances of MTP3 or M3UA.

\section{Rerouting of Registration to the GSM $<->$ CDMA Converting Roaming Hub}

Figures 5 and 6 of Part I of this article [6] show how the registration messages reach the Roaming Hub that is, SEND AUHENTICATION INFO and UPDATE LOCATION (GSM HLR handsets), AUTHENTICATON REQUEST and REGISTER NOTIFICATION (CDMA HLR handsets).

As an example, the E164 numbering plan for Globalstar GSM Europe is:

33640044200-44999 for CDMA handsets

33640000000-19999 for GSM handsets 


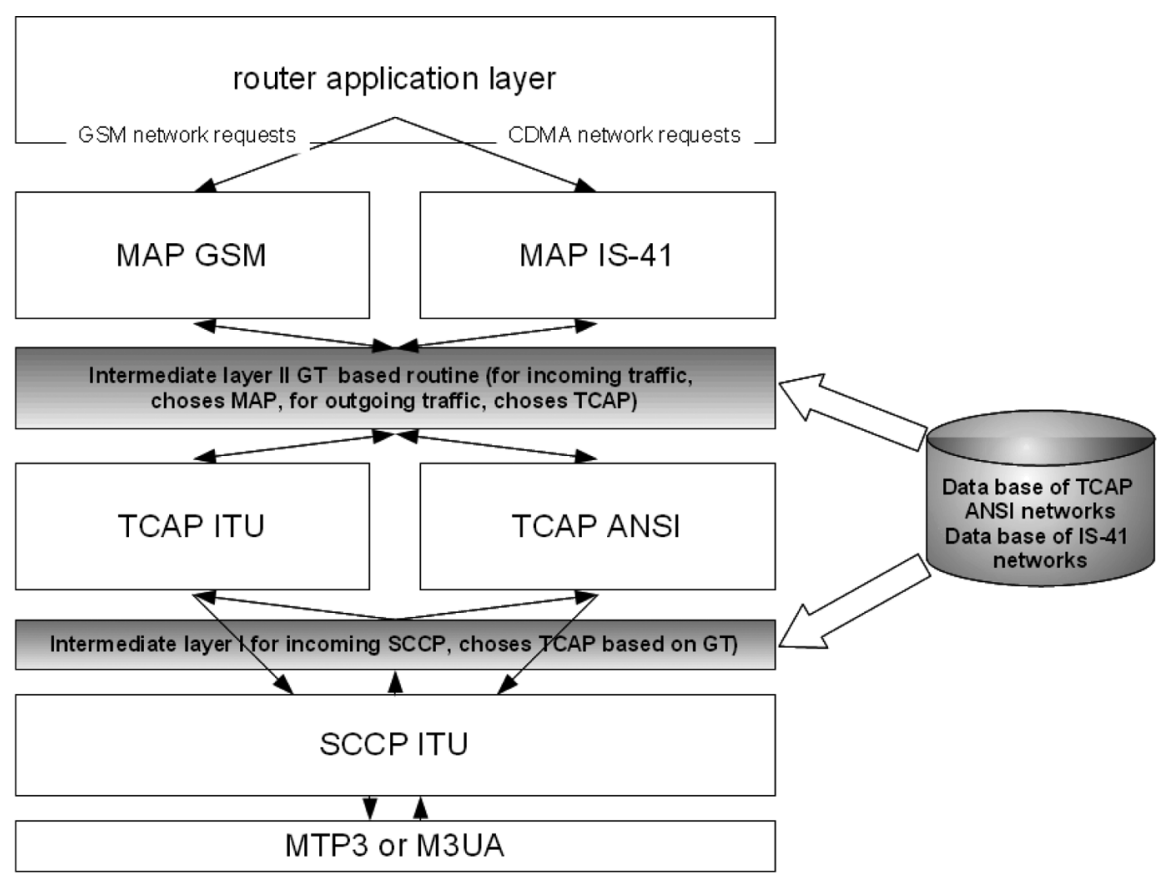

SS7 network with ANSI<-> GSM Point Code conversion

Figure 1 Architecture of a GMS $<->$ CDMA protocol converter.

There are also some ranges for the IMSI (GSM) or MIN (CDMA) assigned to Globalstar Europe.

A sub range of IMSI and MIN is assigned by Globalstar to some planned handsets for the number continuity service. For example:

$+208059990040000-49999$ for the GSM handsets

+40379810000-40379819999 for the CDMA handsets

and as the subscribers subscribe to the service, their IMSI GSM is entered into the Roaming Hub (IMSI-MIN or IMSI-IMSI mapping) depending on whether they have a CDMA handset or a GSM handset.

In the Gateways GMSCs of Globalstar Europe, a new "proxy" HLR for these ranges of numbers is created, that is an E124 routing table which declares the Roaming Hub as their HLR.

GMSC France +208059990040000-49999 $\rightarrow$ 33XXXXXXXX (GT of the single roaming Hub) 


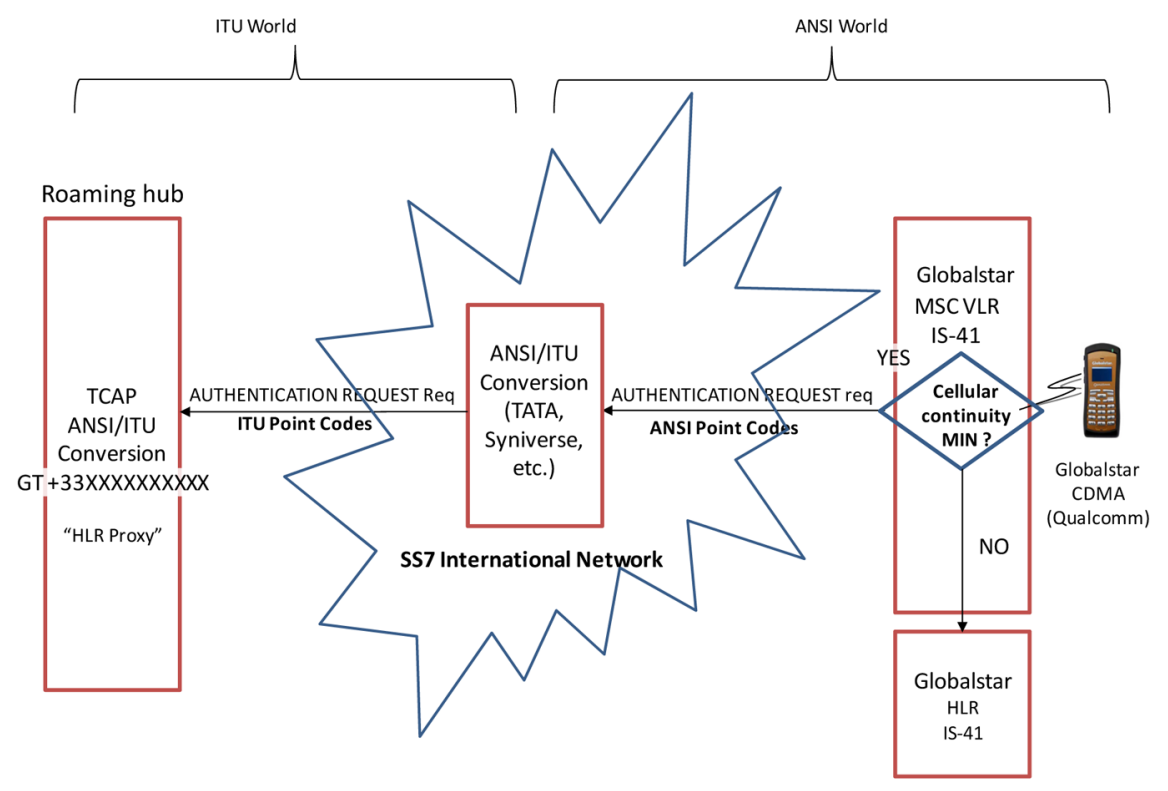

Figure 2 Rerouting to the "HLR proxy".

GMSC USA +40379810000-19999 $\rightarrow$ 33XXXXXXXX (GT of the single roaming $\mathrm{Hub}$ )

This way all the registration messages are forwarded to the Roaming Hub. This is shown in Figure 2 for the rerouting of number continued CDMA Handsets

\section{Details of the GSM $<->$ CDMA Number Continuity Implementation}

As we assume that most readers are quite familiar with MAP GSM, the protocol analyzer used for the traces displays the equivalence GSM when possible in the IS-41 traces below.

\subsection{Authentication VLR $<->$ HLR}

The CDMA handset is powered on.

IS-41 Message Decoding (C) HALYS 2011, 2012

(28): AUTHENTICATION REQUEST (VLR <-> HLR Send Authentication Info 


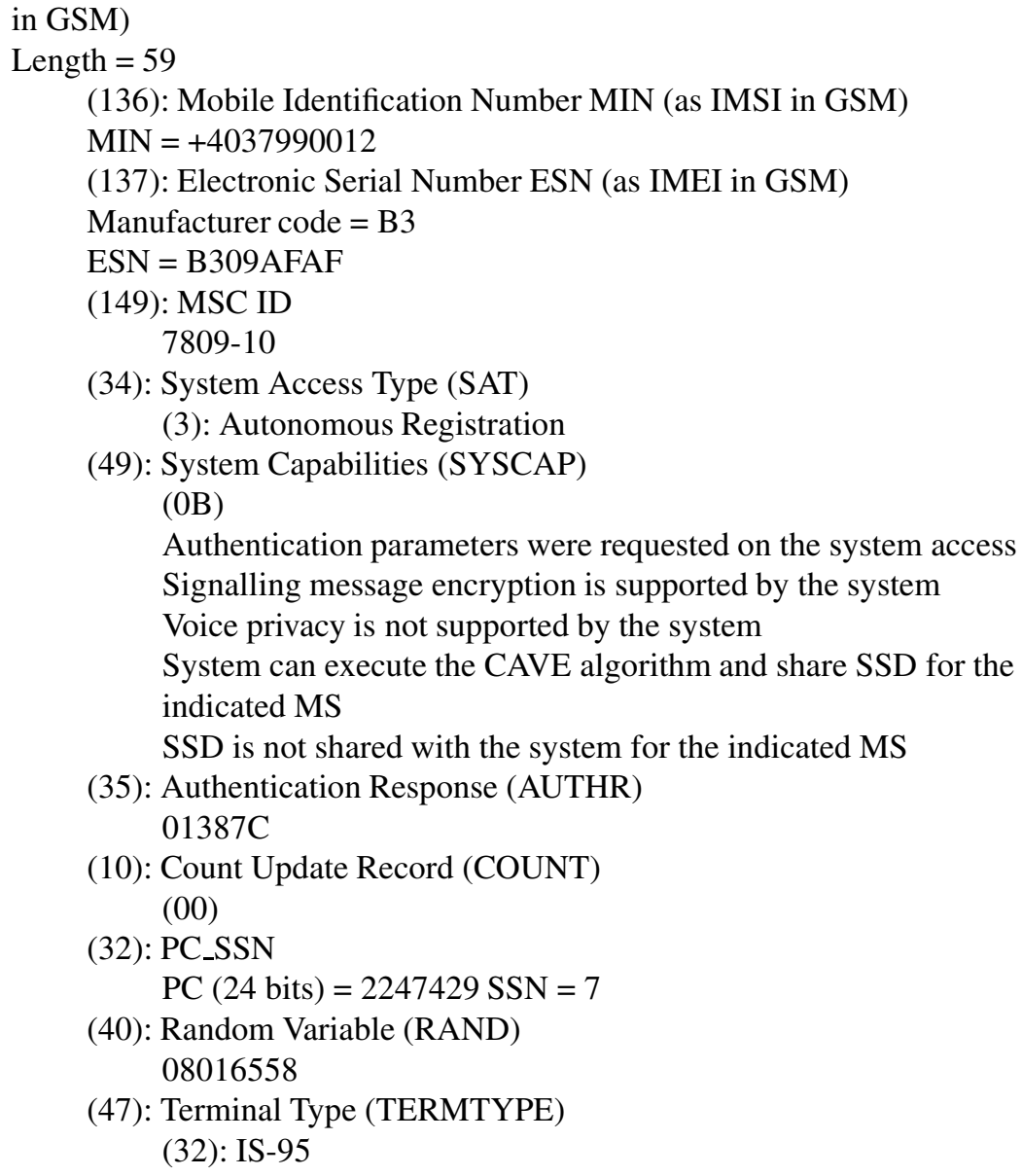

\subsection{Registration}

After a successful answer from the HLR, the subscriber registers and the profile send by the HLR is loaded in the VLR.

\subsubsection{VLR- $>$ HLR Request}

IS-41 Message Decoding (C) HALYS 2011, 2012

(13): REGISTRATION NOTIFICATION (VLR-¿HLR Update Location or HLR-> VLR Insert Subscriber Data in GSM)

Length $=59$ 
4097 (136): Mobile Identification Number MIN (as IMSI in GSM) $\mathrm{MIN}=+4037990012$

(137): Electronic Serial Number ESN (as IMEI in GSM)

Manufacturer code $=\mathrm{B} 3$

$\mathrm{ESN}=\mathrm{B} 309 \mathrm{AFAF}$

(145): Qualification Information code (QUALCODE)

(3): Validation and profile

(150): System My Type Code (MYTYPE)

(16): QUALCOMM

(149): MSC ID

7809-10

(32): PC_SSN

PC $(24$ bits $)=2247429$ SSN $=7$

(104): SMS Address (as Visited MSC GT in GSM)

Type of digit 00

Nature of number 01

International

Presentation allowed

Number is not available

(2): Telephony Numbering E164

(1): $\mathrm{BCD}$

Number of digits 11

$+16139889998$

(53): Extended MSC Identification Number (EXTMSCID)

7809-200

(49): System Capabilities (SYSCAP) (0B)

Authentication parameters were requested on the system access

Signalling message encryption is supported by the system

Voice privacy is not supported by the system

System can execute the CAVE algorithm and share SSD for the indicated MS SSD is not shared with the system for the indicated MS

\subsubsection{HLR->VLR Response with the Subscriber's Profile}

IS-41 Message Decoding (C) HALYS 2011, 2012

(13): REGISTRATION NOTIFICATION (VLR->HLR Update Location or

HLR-> VLR Insert Subscriber Data in GSM)

Length $=65$

(150): System My Type Code (MYTYPE)

(63): Globalstar

(142): Authorization Period (AUTHPER) 
(6): Indefinite,value $=0$

(149): MSC ID

7808-222

(78): Authentication Capabilities

(1): No authentication required

(153): Calling Features Indicator (as Call Forwarding Conditions in

GSM) (CFI)

CFNA CFB CFU CD CNIR CNIP1

(93): Mobile Directory Number MDN (as MSISDN for the SRI_FOR_SM or in the INSERT SUBSCRIBER DATA GSM)

Type of digit 05

Nature of number 31

International

Presentation allowed

Number is not available

(2): Telephony Numbering E164

(1): BCD

Number of digits 10

$\mathrm{MDN}=+4037990012$

(151): Origination Indication (ORIGIND)

(7): International

(152): Termination Restriction Code (TERMRES)

(2): Unrestricted

(48): CDMA Service Option List (IS-737) (CDMALIST)

9F812F0200029F812F0202019F812F020101

The MDN (the subscriber's number which shows in the calls or SMS is then set by the HLR (exactly as in GSM). The test which was done with Globalstar has a small particularity: the MDN Mobile Directory Number (MSISDN in GSM) is the same as the MIN Mobile Identity Number (IMSI in GSM). In general it is not the case with other CDMA networks. Also the CDMA MDN does not include the country code $(+1)$.

In [6, figure 6] (i.e. Part I of this article) showing the number continuity GSM-> Globalstar CDMA, the system will set the MDN sent to the Globalstar VLR $=$ the GSM MSISDN. So when a call or SMS is made with the Globalstar, the GSM number will show.

\subsection{Incoming Call to CDMA Subscriber}

A call is made to the MSISDN of the GSM. The GSM HLR will send a PROVIDE ROAMING NUMBER (which includes the GSM IMSI) to the 


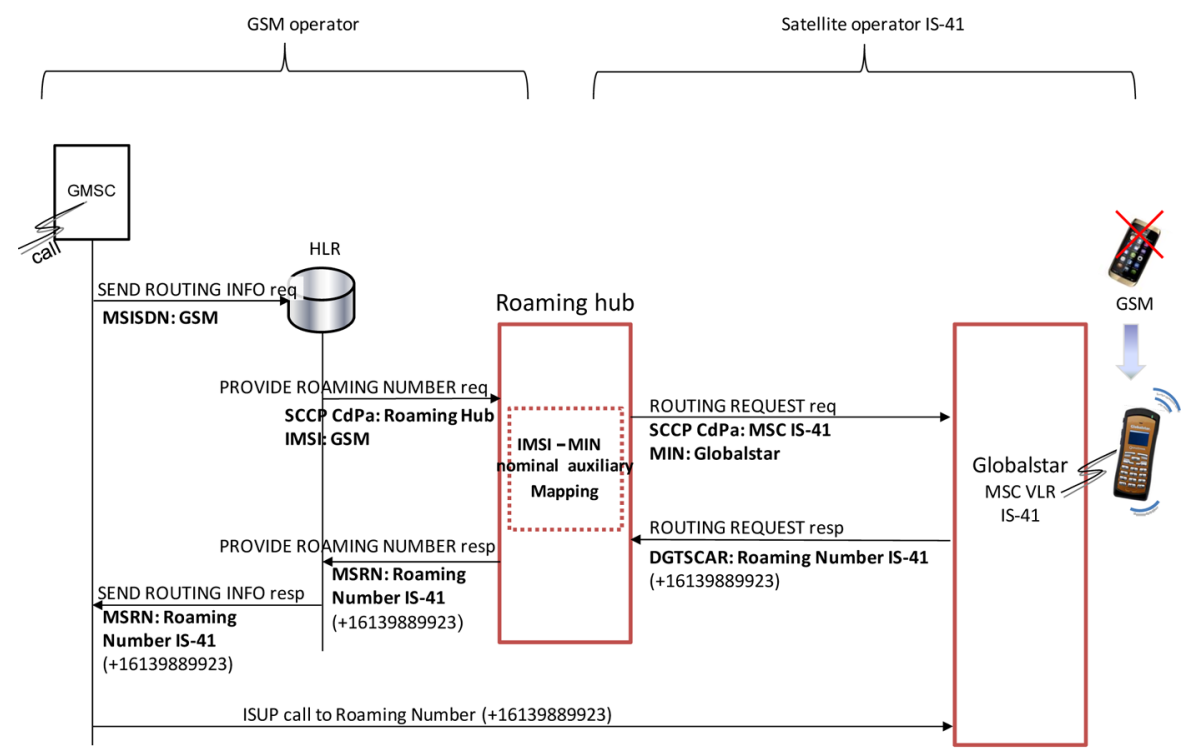

Figure 3 Incoming call to CDMA subscriber.

visited VLR which is the Roaming Hub. The Roaming Hub will map the IMSI to the MIN and will send a ROUTING REQUEST including the MIN (same as the IMSI in GSM). This ROUTING REQUEST also has the GT of the GMSC (which is the GT of the Roaming Hub).

\subsubsection{ROUTING REQUEST Request (Roaming Hub-> VLR)}

IS-41 Message Decoding (C) HALYS 2011, 2012

(16): ROUTING REQUEST (HLR-> VLR Provide Roaming Number GSM)

Length $=52$

(129): Billing ID (BID)

1E81C918587200

(136): Mobile Identification Number MIN (as IMSI in GSM)

$\mathrm{MIN}=+4037990012$

(137): Electronic Serial Number ESN (as IMEI in GSM)

Manufacturer code $=\mathrm{B} 3$

$\mathrm{ESN}=\mathrm{B} 309 \mathrm{AFAF}$

(149): MSC ID

7809-201

(150): System My Type Code (MYTYPE)

(16): QUALCOMM 
(47): CDMA Service Options (IS-737) (CDMASO)

0002

(94): MSC Identification Number (as GT of GMSC in GSM) (MSCIN)

Type of digit 00

Nature of number 31

International

Presentation allowed

Number is not available

(2): Telephony Numbering E164

(1): BCD

Number of digits 11

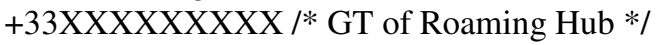

\subsubsection{ROUTING REQUEST Response (VLR->Roaming Hub)}

IS-41 Message Decoding (C) HALYS 2011, 2012

(16): ROUTING REQUEST (HLR-¿VLR Provide Roaming Number GSM)

Length $=37$

(149): MSC ID

7809-10

(129): Billing ID (BID)

1E810A65CF3C00

(132): Digits (called MSISDN from GMSC or MSRN returned by VLR

in GSM) (DGTSCAR)

Type of digit 06

Nature of number 01

International

Presentation allowed

Number is not available

(2): Telephony Numbering E164

(1): BCD

Number of digits 11

$+16139889923 / *$ Roaming Number allocated by the VLR IS-41 and returned to the Roaming Hub */

(32): PC_SSN

$\mathrm{PC}(24$ bits $)=2247429 \mathrm{SSN}=7$

The Roaming Hub will give +16139889923 in the GSM PROVIDE ROAMING NUMBER Confirmation. As a result the GSM GMSC will call +16139889923 directly and the IS-41 network will receive the same Mobile Terminated Calls charges. 


\subsubsection{Call Forwarding IS-41 for Unsuccessful Mobile Terminated Calls}

This does not work like GSM. In GSM the VLR profile contains "conditional call forwarding" informations for call busy, no response, not reachable. There is no such thing in IS-41, the profile returned by the HLR in the REGISTER NOTIFICATION result returned does not have it. When the incoming call of Figure 3 fails, the VLR IS-41 sends to the HLR a TRANSFER TO NUMBER REQUEST with the "Redirection Reason", asking for instructions.

IS-41 Message Decoding (c) HALYS 2011, 2012

(23): TRANSFER TO NUMBER REQUEST (VLR->HLR-> VLR) (the VLR tells the result of a MT call and receives a redirection number)

Length $=22$

(136): Mobile Identification Number MIN (as IMSI in GSM)

$\mathrm{MIN}=+4037990012$

(137): Electronic Serial Number ESN (as IMEI in GSM)

Manufacturer code $=\mathrm{B} 3$

$\mathrm{ESN}=\mathrm{B} 309 \mathrm{AFAF}$

(150): System My Type Code (MYTYPE)

(16): QUALCOMM

(147): Redirection Reason

(4): No Page Response

The HLR responds by sending a "Redirecting Number" which could be the GSM VMS number, which is then called by the VLR. For the number continuity service, the Roaming Hub has extracted the GSM conditional call forwarding numbers from the INSERT SIBSCRIBER DATA and uses them to create the TRANSFER TO NUMBER Response sent to the VLR.

IS-41 Message Decoding (C) HALYS 2011, 2012

(23): TRANSFER TO NUMBER REQUEST (VLR->HLR->VLR) (the VLR tells the result of a MT call and receives a redirection number)

Length $=36$

(132): Digits (called MSISDN from GMSC or MSRN returned by VLR

in GSM) (DGTSCAR)

Type of digit 01

Nature of number 00

National

Presentation allowed

Number is not available

(2): Telephony Numbering E164

(1): BCD

Number of digits 10 


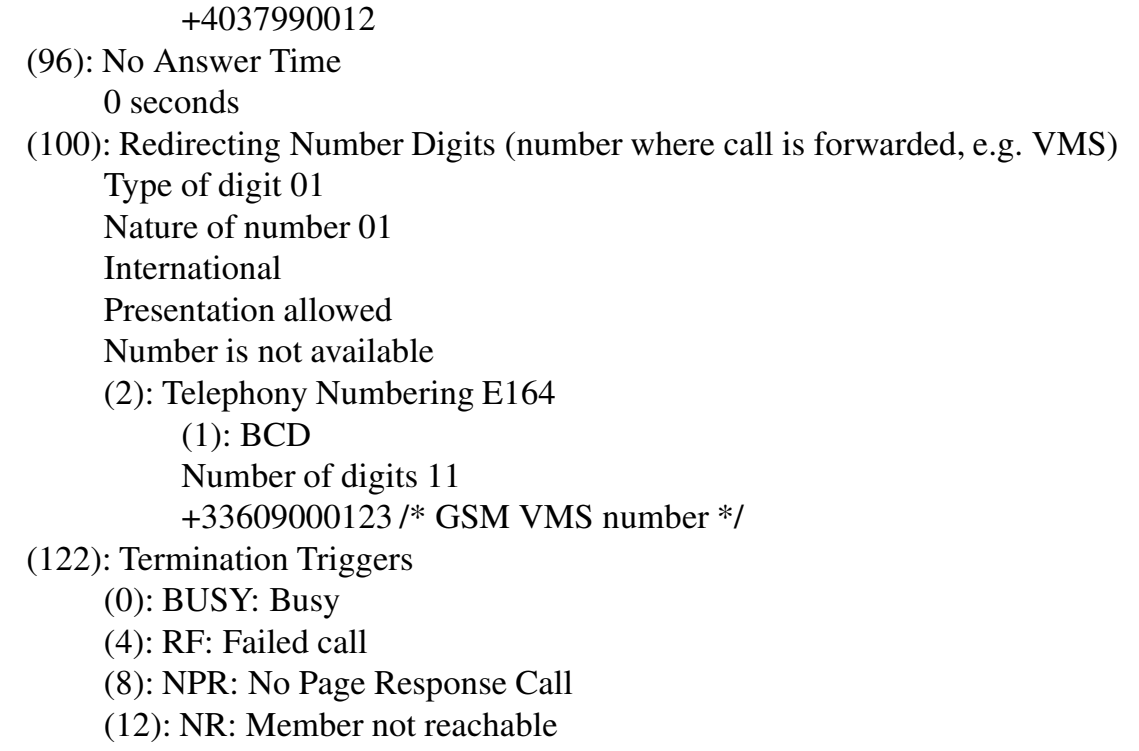

The VLR will then forward the voice call to +33609000123 as shown in Figure 3.

\subsection{Deregistration}

This occurs when a subscriber powers down the handset. A signaling message is sent from the VLR to the HLR to deregister the handset.

IS-41 Message Decoding (C) HALYS 2011, 2012

(22): MS INACTIVE (VLR-> HLR MS Purge GSM)

Length $=16$

(136): Mobile Identification Number MIN (as IMSI in GSM)

$\mathrm{MIN}=+4037990012$

(137): Electronic Serial Number ESN (as IMEI in GSM)

Manufacturer code $=\mathrm{B} 3$

$\mathrm{ESN}=\mathrm{B} 309 \mathrm{AFAF}$

\subsection{Incoming SMS-MT to CDMA Subscriber}

\subsubsection{The Local or Foreign SMSC Asks the HLR for the Visited MSC and MIN}

This is the case of a SMSC, not the "number continuity" case which is simpler and does not need to interrogate the HLR IS-41. 


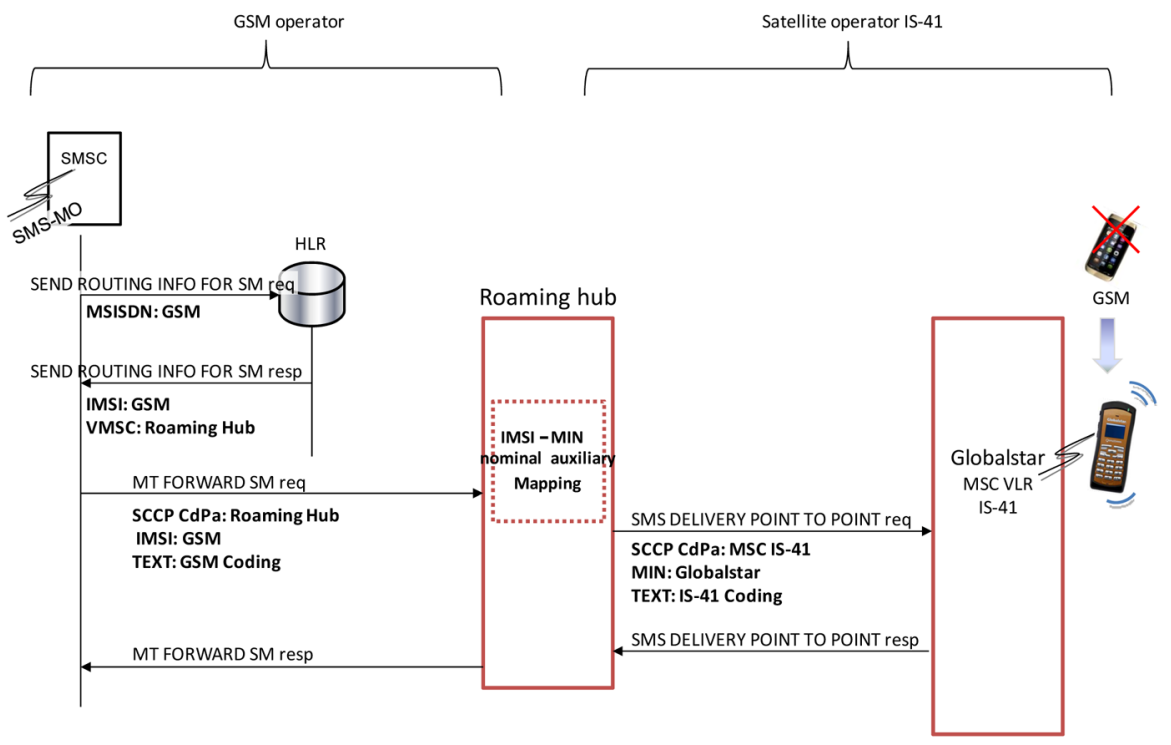

Figure 4 Incoming SMS-MT to CDMA subscriber.

The local or foreign SMSC uses the known public number MDN of the subscriber same as the MSIDN in a SRI_FOR_SM and gets the Visited MSC GT and the MIN (same as IMSI) which will be used in the SMS DELIVERY POINT TO POINT (same as FWD_SM_MT in GSM).

IS-41 Message Decoding (C) HALYS 2011, 2012

(55): SMS REQUEST (SMSC $<->$ HLR Send Routing Information for SM in GSM) Length $=24$

(109): SMS Notification indicator (demand of a HLR alert by the SMSC)

(SMSNOTIND)

Notify when available (01)

(116): SMS Teleservice Identifier (IS-637) (SMSTID)

CDMA Number Messaging Teleservice (1002)

(93): Mobile Directory Number MDN (as MSISDN for the SRI_FOR_SM or in the INSERT SUBSCRIBER DATA GSM)

Type of digit 05

Nature of number 31

International

Presentation allowed

Number is not available

(2): Telephony Numbering E164

(1): BCD 


$$
\begin{aligned}
& \text { Number of digits } 10 \\
& \text { MDN }=+4037990012
\end{aligned}
$$

This is the response of the HLR including the MIN. This is particular (Globalstar) and they use a MDN (the MSISDN in GSM) equal to the MIN (the IMSI in GSM).

IS-41 Message Decoding (C) HALYS 2011, 2012

(55): SMS REQUEST (SMSC <-> HLR Send Routing Information for SM in GSM) Length $=29$

(137): Electronic Serial Number ESN (as IMEI in GSM)

Manufacturer code $=\mathrm{B} 3$

$\mathrm{ESN}=\mathrm{B} 309 \mathrm{AFAF}$

(104): SMS Address (as Visited MSC GT in GSM)

Type of digit 05

Nature of number 31

International

Presentation allowed

Number is not available

(1): ISDN Numbering Plan

(2): IA5 International Alphabet 5

Number of digits 11

$+16139889998$

(136): Mobile Identification Number MIN (as IMSI in GSM)

$\mathrm{MIN}=+4037990012$

\subsection{The SMSC Sends the SMS to the Visited MSC}

For number continuity the Roaming Hub does not need to interrogate the HLR IS-41 because it already knows the MIN and the visited VLR IS-41. It will send the SMS DELIVERY POINT TO POINT directly.

IS-41 Message Decoding (C) HALYS 2011, 2012

(53): SMS DELIVERY POINT TO POINT (SMSC->MSC SMS-MO or MSC->SMSC SMS-MT Forward Short Message GSM)

Length $=188$

(136): Mobile Identification Number MIN (as IMSI in GSM) $\mathrm{MIN}=+4037990012$

(137): Electronic Serial Number ESN (as IMEI in GSM)

Manufacturer code $=\mathrm{B} 3$

$\mathrm{ESN}=\mathrm{B} 309 \mathrm{AFAF}$

(105): SMS Bearer Data

MESSAGE_ID: 
message type $=01($ Deliver $($ mobile terminated only $))$

message_ID: 0AD7

USER_DATA

Subparam length $=16$

Msg_Encoding $=02(7$ bits ASCII $)$

Num $7 b$ characters $=16$

User_Data $=$ Globalstar

NUMBER OF MSGs IN VMS: 12

LANGUAGE_INDICATOR: 02 (French)

MESSAGE CENTER TIMESTAMP: year $=2011$ month $=12$ day $=$ 10 hour $=6 \mathrm{~min}=45 \mathrm{sec}=32$

VALIDITY PERIOD (absolute format): year $=2011$ month $=12$

day $=14$ hour $=10 \mathrm{~min}=0 \mathrm{sec}=0$

VALIDITY PERIOD (relative format) $=85$ (that is 25800 seconds)

ALERT ON MESSAGE DELIVERY: Use high priority alert MESSAGE DISPLAY MODE (1): Mobile default setting: as predefined in the MS

REPLY OPTION:

User Ack (if this is SMS-MT) = Positive (manual) User ACK

requested from the recipient user

Delivery Ack (if this is SMS-M0) $=$ No Delivery ACK requested

from the recipient

PRIORITY INDICATOR (2): Urgent

PRIVACY INDICATOR (3): Secret

DEFERRED DELIVERY TIME (absolute format): year $=2012$

month $=1$ day $=6$ hour $=23 \mathrm{~min}=59 \mathrm{sec}=0$

DEFERRED DELIVERY TIME (relative format) $=84$ (that is

25500 seconds)

USER RESPONSE CODE (predefined by SMSC for the SMSack)

$=33$

CALL BACK NUMBER

Digit Mode $=00($ DTMF $(4$ bits BCD $))$

Num_Fields $=13$

Call Back Number $=1234567890 \mathrm{ABC}$

CALL BACK NUMBER

Digit Mode $=01($ ASCII $(8$ bits $))$

Numbering type $=01$

Numbering plan $=02$

Num_Fields $=6$

Call Back Number $=1234 \mathrm{AB}$

(116): SMS Teleservice Identifier (IS-637) (SMSTID)

CDMA Voice mail notification (4099) 
(109): SMS Notification indicator (demand of a HLR alert by the SMSC) (SMSNOTIND)

Notify when available (01)

\subsubsection{The HLR Alerts the SMSC When Subscriber Becomes Reachable}

This is like ALERT SERVICE CENTRE in GSM. The SMSC will retry.

IS-41 Message Decoding (C) HALYS 2011, 2012

(54): SMS NOTIFICATION (HLR->SMSC Alert SC GSM)

Length $=29$

(137): Electronic Serial Number ESN (as IMEI in GSM)

Manufacturer code $=\mathrm{B} 3$

$\mathrm{ESN}=\mathrm{B} 309 \mathrm{AFAF}$

(136): Mobile Identification Number MIN (as IMSI in GSM)

MIN $=+4037990012$

(104): SMS Address (as Visited MSC GT in GSM)

Type of digit 05

Nature of number 31

International

Presentation allowed

Number is not available

(1): ISDN Numbering Plan

(2): IA5 International Alphabet 5

Number of digits 11

$+16139889998$

\subsection{Internet Data Services for CDMA-> GSM Number Continuity}

The likely usage is a GSM usage in a VPLMN. The Local Break-Out provides a simple solution so that the CDMA subscriber can have the internet access while using a GSM handset. But the GTP Protocol is common for GSM and CDMA2000, so that the PDP Context can also be established with the HPLMN CDMA GGSN [5].

\section{CDMA $<->$ GSM Number Continuity Service}

This is a real case with major networks, such as SPRINT(CDMA) in the USA which provide the international roaming services to their subscribers with a dual standard handset CDMA plus a GSM SIM card from a "sponsor" and a Roaming Hub supplier providing the conversion. This is not a new idea 


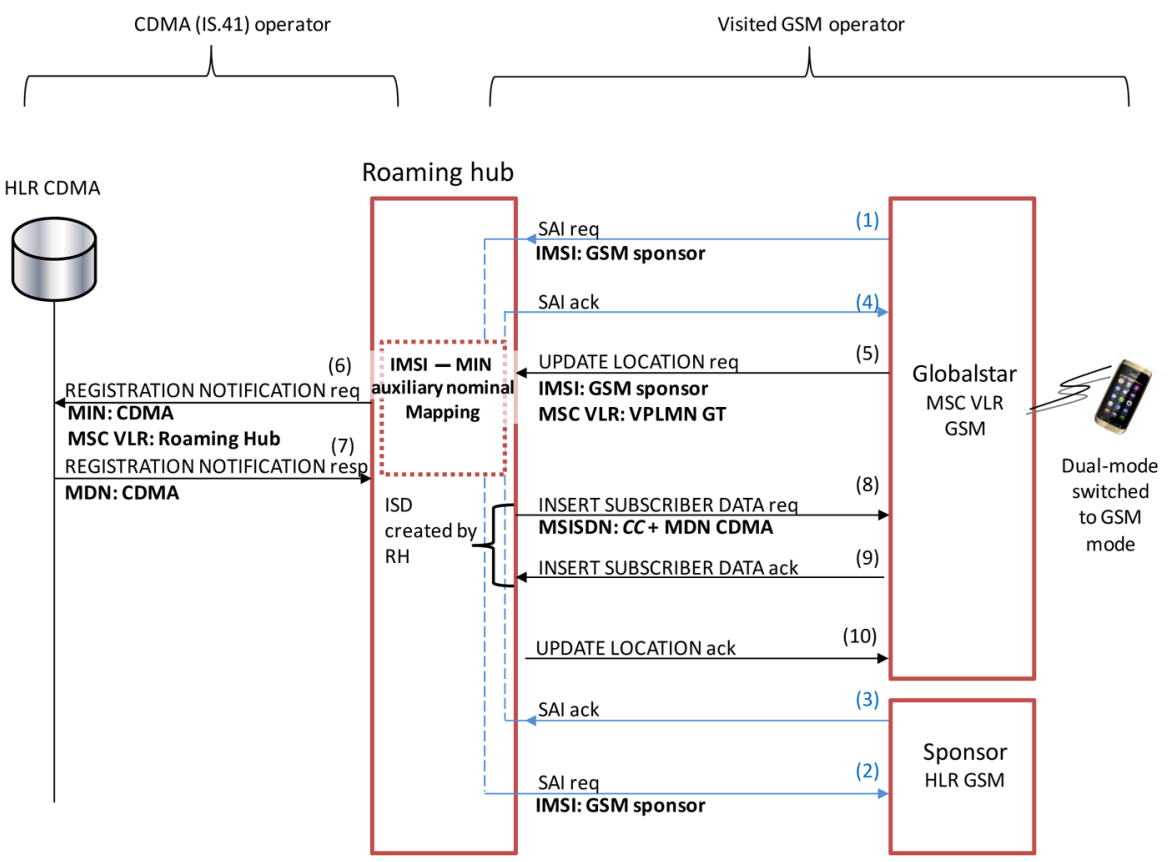

Figure 5 CDMA $<->$ GSM registration with a Roaming Hub.

and the service was offered since 2003 with a separate GSM handset provide by a sponsor. It is more practical with dual standard handsets including the latest Iphone. When he arrives in Europe for example, the user selects the GSM mode or a use a separate GSM handset, the number continuity service is provided with the IS-41 HLR thanks to the two-way conversion in the Roaming Hub as shown by Figure 5. Compare with figures 5 and 6 of Part I [6].

In IS-41 there is no equivalent of MAP GSM INSERT SUBSCRIBER DATA, the MDN of the user (equivalent of the MSISDN) is sent by the HLR CDMA in the REGISTRATION NOTIFICATION resp. (7). The Roaming Hub creates an INSERT SUBSCRIBER DATA req (8) which contains the MSISDN to be used in GSM roaming. Figure 5 shows that

$$
\text { MSISDN }=\mathrm{CC}+\mathrm{MDNCDMA}
$$

$\mathrm{CC}$ would be +1 in the case of a US roamer, which is added by the Roaming Hub because in many cases the MDN does not include the country code CC. 


\section{References}

[1] TIA/EIA-41-D. Number Radiotelecommunications Intersystem Operations, 1997. (Main description of the IS-41 protocol with the tables of operation and parameter codes.)

[2] TIA/EIA-637-A. Short Message Service for Spread Spectrum Systems, 1999. (Complement to [10.1] for the SMS service. Gives all the details and tables to implement the SMS service.)

[3] TIA/EIA-737. (Describes additional parameters to [1].)

[4] TIA/EIA-707-D. Based Network Enhancements for CDMA Packet Data Service (CPDS). 3GPP2 N.S0029 V1.0.0, June 2002.

[5] IFAST\#24/2004.10.04/07. CDMA Packet Data Roaming eXchange Guidelines.

[6] A. Henry-Labordère. The number continuity service: Part I, GSM $<->$ satellite phone. Journal of Cyber Security and Mobility, 1(4):349-376, 2013.

\section{Biography}

Arnaud Henry-Labordère is a graduate engineer from Ecole Centrale de Paris (1966), Ph.D. (Mathematics, USA, 1968). He was professor of Operations Research at Ecole Nationale des Ponts et Chaussées during 25 years, as well as at Ecole Nationale des Mines de Paris. He is currently Visiting Professor at Prism-CNRS. He started at IBM research (1967) and founded three companies: FERMA (voice mail systems in 1983), Nilcom (first SMS network in 1999) and currently Halys (telecom equipments). He is the author of eight books (six in maths, two in telecoms) and has been granted 85 patents. 